\title{
Emission characteristics of polycyclic aromatic hydrocarbons and nitro-polycyclic aromatic hydrocarbons from diesel trucks based on on-road measurements
}

Xinyue Cao, Xuewei Hao, Xianbao Shen, Xi Jiang, Bobo Wu, Zhiliang Yao ${ }^{1}$

School of Food and Chemical Engineering, Beijing Technology and Business University, Beijing 100048, China

\begin{abstract}
Polycyclic aromatic hydrocarbon (PAH) and nitro-polycyclic aromatic hydrocarbon (NPAH) emissions from 18 diesel trucks of different sizes and with different emission standards were tested in Beijing using a portable emission measurement system (PEMS). Both the gaseous- and particulate-phase PAHs and NPAHs were quantified by high-performance liquid chromatography (HPLC) in the laboratory. The emission factors (EFs) of the total PAHs from light-duty diesel trucks (LDDTs), medium-duty diesel trucks (MDDTs) and heavy-duty diesel trucks (HDDTs) were $82229.11 \pm 41906.06,52867.43 \pm 18946.47$ and $93837.35 \pm 32193.14 \mu \mathrm{g} / \mathrm{km}$, respectively, much higher than the respective values of total NPAHs from their counterpart vehicles. The gaseous phase had an important contribution to the total PAHs and NPAHs, with a share rate of approximately $69 \%$ and $97 \%$ on average, respectively. The driving cycle had important impacts on the emissions of PAHs and NPAHs, especially for LDDTs and HDDTs. Higher emissions of PAHs and NPAHs were detected on non-highway roads compared to that on highways for these two types of vehicles. Compared to the results of different studies, the difference in the EFs of PAHs and NPAHs can reach several orders of magnitudes, which would introduce errors in the development of an emission inventory of PAHs and NPAHs.
\end{abstract}

Keywords: PAHs; NPAHs; diesel vehicle; emission factors; PEMS

${ }^{1}$ Z. Yao. Telephone: +86 10-68984641. Email: yaozhl@ th.btbu.edu.cn. 


\section{Introduction}

Polycyclic aromatic hydrocarbons (PAHs) and nitro-polycyclic aromatic hydrocarbons (NPAHs) have attracted considerable concern due to their toxicity, mutagenicity and carcinogenicity (Banford et al., 2003; Albinet et al., 2007; Casal et al., 2014). NPAHs are derived from the reaction of the parent PAHs with hydroxyl $(\mathrm{OH})$ and nitrate $\left(\mathrm{NO}_{3}\right)$ radicals in the presence of NOx or through nitration during combustion processes (Tsapakis et al., 2007), which have toxicological significance even if present at much lower concentrations than their parent compound (Grimmer et al., 1987; IARC, 1989; Durant et al., 1996; Shen et al., 2012).

Both PAHs and NPAHs can form through the incomplete combustion of organic matter such as coal, oil and wood (Chetwittayachan et al., 2002; Thuy et al., 2012). Shen et al. (2013) estimated that on-road motor vehicles were the fourth important major source and contributed approximately $12.8 \%$ of the global total annual atmospheric emissions of 16 PAHs in 2007. In urban cities, vehicle exhaust is one of the most important sources of PAHs and NPAHs, especially diesel engine exhaust (Wada et al., 2001; Chen et al. 2004; Chang et al., 2006; Ravindra et al., 2006; Logan 2007). Zhang et al. (2009) found that motor vehicles and heating were the most important sources of PAHs in Beijing. Zhou et al. (2005) reported that the total concentration of 17 PAHs in an urban area was 1.1-6.6 times higher than that measured in a suburban area of Beijing. Fromme et al. (2004) inferred that motor vehicles were the main source of PAHs in urban residential areas of Berlin, Germany. Wada et al. (2001) found that the concentration of NPAHs in particulate matter was positively correlated with the traffic volume and concluded that diesel vehicles were the main source of NPAHs in the atmosphere in Nagasaki, Japan. However, the contribution of motor vehicles to global PAH emissions is less than that of biomass burning and wildfires (Shen et al. 2013), and the relative contribution of PAHs from motor vehicles to the human exposure risk is much higher than its contribution to the emissions themselves because motor vehicle emissions occur mostly in urban areas where population densities are much higher than the global average. The inhalation intake fraction, defined as the fraction of the emission that is eventually inhaled, of benzo(a)pyrene from motor vehicles in China was estimated to be 4 times greater than the mean inhalation intake fraction from all other sources (Zhang et al., 2009).

In recent decades, many researchers have conducted studies of the emission characteristics of 
PAHs and NPAHs from motor vehicles via chassis dynamometer tests (de Abrantes et al. 2004; Bikas et al. 2007; Karavalakis et al. 2010; Huang et al., 2013; Hu et al., 2013) and tunnel experiments (Benner et al., 1989; Fraser et al., 1998; Ho et al., 2009; Chen et al., 2013). The emission factors (EFs), gas-particle partitioning, isomer distribution, impact factors, etc. for PAHs and NPAHs from motor vehicles have been widely studied. However, the existing studies mainly focused on PAHs, especially on particulate-phase PAHs, which have been investigated for decades (Heeb et al., 2010). Studies on NPAHs and gaseous-phase PAHs are relatively few. More research on the emission characteristics of NPAHs and gaseous-phase PAHs is necessary.

In recent years, with the development of the portable emission measurement system (PEMS), more on-road studies have been carried out on vehicular emissions using this technique. The emission characteristics of $\mathrm{CO}_{2}, \mathrm{CO}, \mathrm{HC}, \mathrm{PM}$, and the chemical constituents of PM and VOCs have been widely studied using this method because the results reflect real emissions from vehicles. However, studies reporting the on-road emissions of PAHs and NPAHs using the PEMS are very limited. Shah et al.(2005) presented the emission rates of PAHs from on-road emissions testing of nine heavy-duty diesel (HDD) vehicles tested using CE-CERT's Mobile Emissions Laboratory (MEL) over the California Air Resources Board (ARB) Four-Phase Cycle. This is the only paper related to the on-road emission characteristics of PAHs or NPAHs published to date.

In this study, the main purpose is to understand the real emissions of gaseous- and particulate-phase PAHs and NPAHs and the impact of several factors on the emissions of PAHs and NPAHs. To achieve this objective, PAH and NPAH emissions from nine China III and nine China IV diesel trucks of different sizes (light, medium, and heavy duty) in Beijing were tested using a PEMS. Both the gaseous- and particulate-phase PAHs and NPAHs were collected and then quantified by high-performance liquid chromatography (HPLC) in the laboratory. Based on the measured data, the EFs and distributions of PAHs and NPAHs and the impact factors were characterized. The results of this study will be helpful for understanding the real emission levels of PAHs and NPAHs from diesel trucks on roads.

\section{Experimental Section}

\subsection{Portable Emission Measurement System (PEMS)}


A combined PEMS was developed based on our previous studies (Yao et al., 2011; Huo et al., 2012) that mainly consisted of a Semtech-DS (Sensors Inc., USA), exhaust flow meter (EFM-2, Sensors Inc., USA), micro-proportional sampling system (MPS, Sensors Inc., USA) and PAH-collection unit. The Semtech-DS was used to analyze the emissions of gaseous pollutants at a $1 \mathrm{sec}$ resolution. The EFM-2 was employed to measure the instantaneous mass flow of the exhaust, which can be used to calculate the EF of the pollutants. The MPS was applied to dilute the exhaust sample before it was passed through the PAH-collection unit. The dilution ratios were approximately 8:1. The PAH-collection unit included quartz filters (QFs, $47 \mathrm{~mm}$ in diameter) followed by two-stage polyurethane foam (PUF) cartridges. In addition, a GPS unit was used to record the instantaneous speed and driving distance. More information related to the PEMS can be found in our previous studies (Yao et al., 2015a; Wu et al., 2016).

\subsection{On-Road Experiments}

The on-road experiments were carried out in southwest Beijing. In total, 18 vehicles, including six types of diesel trucks of different sizes (light, medium and heavy) and different emission standards (China 3 and China 4), were tested, and three vehicles were tested for each type of truck. For all the tested China IV light-duty diesel trucks (LDDTs) and medium-duty diesel trucks (MDDTs), particulate oxidation catalyst (POC), diesel oxidation catalyst (DOC) and exhaust gas recirculation (EGR) control devices were installed. Selected catalytic reduction (SCR) control devices were installed on all the tested China 4 heavy-duty diesel trucks (HDDTs). No after-treatment control device was installed on any of the China 3 diesel trucks tested. All of the test vehicles have manual transmissions. Detailed information on the tested trucks is listed in Table S1 in the Supplemental Materials. The measurements were taken when the test vehicles were driven repeatedly on a predesigned test route. The length of the test route was $47 \mathrm{~km}$ for the LDDTs and MDDTs and $63 \mathrm{~km}$ for the HDDTs. Both of test routes included highway and non-highway roads. In addition, the diesel trucks were fueled with commercially available diesel fuel from Beijing that met the $10 \mathrm{mg} / \mathrm{kg}$ sulfur limit of the Chinese phase V diesel standard. During the measurements, the trucks were not in service and were under hot stabilized conditions, and an assistant was onboard the trucks to check the emissions equipment. The total weight of the emissions equipment was approximately $200 \mathrm{~kg}$. More information on the test route and measurements can be found in our previous studies (Yao et al. 2015a; Wu et al., 2016). 


\subsection{Sample Collection}

Particle-phase PAHs and NPAHs were collected on a $47 \mathrm{~mm}$ QF (2500QAT-UP, PALL Corp., USA) the QFs. Gas-phase PAHs were sampled by two-stage PUF cartridges. The flow rate of the sampling was controlled at approximately $3 \mathrm{~L} / \mathrm{min}$, and the flow meter used in this work was calibrated with a BIOS Defender 530 (Drycal Technology Inc., USA) before each test. The samples were collected when the tested trucks were driven on highway and non-highway roads, respectively. Each type of road was tested twice. The sampling time for each test was approximately 30-40 min. After sampling, the samples were stored at $-10{ }^{\circ} \mathrm{C}$ before analysis. Before sampling, a PUF was pre-cleaned in an ultrasonic cleaner with dichloromethane three times, with each time lasting 30 minutes. The QFs were pre-combusted at $550{ }^{\circ} \mathrm{C}$ for $12 \mathrm{~h}$ in a muffle furnace.

\subsection{PAH and NPAH Analysis}

Sample pretreatment followed standardized internal procedures (Yao et al., 2015b; Hao et al., 2016). Firstly, the samples were ultrasonically extracted for $30 \mathrm{~min}$ with $10 \mu \mathrm{L}$ of a mixed solvent (naphthalene-d8, acenaphthene-d10, phenanthrene-d10, chrysene-d12, and perylene-d12) and dichloromethane $(150 \mathrm{~mL}$ for $\mathrm{QF}$ and $15 \mathrm{~mL}$ for $\mathrm{PUF})$ three times. The extracts were then concentrated to approximately $1 \mathrm{~mL}$ and subsequently solvent-exchanged to hexane. The concentrated extracts were then transferred to a silica/alumina chromatography column $(20 \mathrm{~cm}$ of silica gel and $10 \mathrm{~cm}$ of alumina) and eluted with $50 \mathrm{~mL}$ of $\mathrm{n}$-hexane and $50 \mathrm{~mL}$ of dichloromethane/hexane (1:1). The eluate was concentrated to $1 \mathrm{~mL}$ and converted to an acetonitrile solution.

The solutions were analyzed for PAHs and NPAHs using an HPLC system (Agilent 1200, Santa Clara, CA, USA) equipped with a UV detector (Agilent G1314A, Santa Clara, CA, USA), detected at $220 \mathrm{~nm}, 230 \mathrm{~nm}, 254 \mathrm{~nm}$ and $290 \mathrm{~nm}$, and an Agilent HC-C18 chromatographic column $(5 \mu \mathrm{m}$, $250 \times 4.6 \mathrm{~mm}^{2}$ ) (Agilent, USA) operated at $30^{\circ} \mathrm{C}$. A linear binary gradient composed of acetonitrile (A) and water (B) was employed using the following scheme: 65\% A for 27 min, $65-100 \%$ A for 14 min, and maintenance of isocratic conditions $(100 \%$ A) for $5 \mathrm{~min}$. When the initial conditions were restored, the column was re-equilibrated for $8 \mathrm{~min}$. The flow rate of the eluent was controlled at 1.2 $\mathrm{mL} / \mathrm{min}$. Compounds were quantified using external calibration methods.

A mixed standard solution of 16 PAHs was used for the calculation of the PAHs and included naphthalene (Nap, two-ring), acenaphthylene (Acl, three-ring), fluorene (Flr, three-ring), 
acenaphthene (Acp, three-ring), phenanthrene (Phe, three-ring), anthracene (Ant, three-ring), fluoranthene (Flu, four-ring), pyrene (Pyr, four-ring), chrysene (Chr, four-ring), benzo[a]anthracene (BaA, four-ring), benzo[b]fluoranthene (BbF, five-ring), benzo[k]fluoranthene (BkF, five-ring), benzo[a]pyrene (BaP, five-ring), dibenzo[a,h]pyrene (DBahA, five-ring), benzo[g,h,i]perylene (BghiP, six-ring), and indeno[1,2,3-cd]pyrene (IcP, six-ring) (o2si Co., USA). The recovery efficiencies of the 16 PAH compounds ranged from $60.32 \%$ to $123.00 \%$, with an average value of 93.90\%. The detection limits of the $16 \mathrm{PAHs}$ ranged from 0.38 to $9.09 \mathrm{ng} / \mathrm{mL}$ at a signal-to-noise ratio of 3. The PAH concentrations were not detectable in field blanks or laboratory blanks.

The NPAHs were quantified using a mixed standard solution of 9 NPAHs: 5-nitroacenaphthene (5-NAcp, three-ring), 9-nitroanthracene (9-NAnt, three-ring), 3-nitrophenanthrene (3-NPhe, three-ring), 1,3-nitropyrene (1,3-NPyr, four-ring), 1-nitropyrene (1-NPyr, four-ring), 3-nitrofluoranthene (3-NFlu, four-ring), 6-nitrochrysene (6-NChr, four-ring), 7-nitrobenz[a]anthracene (7-NBaA, four-ring), and 6-nitrobenz[a]pyrene (6-NBaP, five-ring) (o2si Co., USA). The recovery efficiencies of the 9 NPAH compounds ranged from $68.37 \%$ to $107.46 \%$, with an average value of $90.22 \%$. The detection limits of the 9 NPAHs ranged from 0.93 to 8.28 $\mathrm{ng} / \mathrm{mL}$ at a signal-to-noise ratio of 3 . The NPAH concentrations were not detectable in field blanks or laboratory blanks.

\section{Results and Discussion}

\subsection{Emission Factors (EFs)}

The EFs of the 16 PAHs and nine NPAHs based on the mileage traveled were calculated by the vehicle type, as shown in Table S2 in the Supplemental Materials. Figure 1 summarizes the sum of the gaseous-phase, particulate-phase, and total PAHs and NPAHs for the different types of vehicles on average. The PAH and NPAH EFs varied with the vehicle type. However, no clear trends were found with changes in the vehicular weight from light to heavy or in the emission standard from China 3 to China 4. Except the MDDTs, the EFs of the PAHs and NPAHs did not decrease with the change from the China 3 to China 4 emission standard for LDDTs and HDDTs. The EFs also did not present a trend with the change in the vehicle gross weight from light-duty to heavy-duty trucks. We cannot explain why the EFs of the PAHs and NPAHs from MDDTs were relatively lower compared with the LDDTs and HDDTs, and similar trends were also found for the VOC EFs reported in our 
previous study (Yao et al., 2015c). Further investigation of emissions from MDDTs should be conducted in the future to explore this phenomenon.

Table 1 lists the mean EFs of the isomers, the sums of the PAHs and NPAHs and the standard deviations for the LDDTs, MDDTs and HDDTs tested in this study, as well as the results for diesel vehicles reported in the literature. The EFs of the total PAHs for LDDTs, MDDTs and HDDTs were 82229.11 $\pm 41906.06,52867.43 \pm 18946.47$ and $93837.35 \pm 32193.14 \mu \mathrm{g} / \mathrm{km}$, respectively, much higher than the respective values of total NPAHs from their counterpart vehicles, respectively.

Table 1 Mean emission factors and standard deviations of PAHs and NPAHs for the LDDTs, MDDTs and HDDTs tested in this study and the results for diesel vehicles reported in the literature.

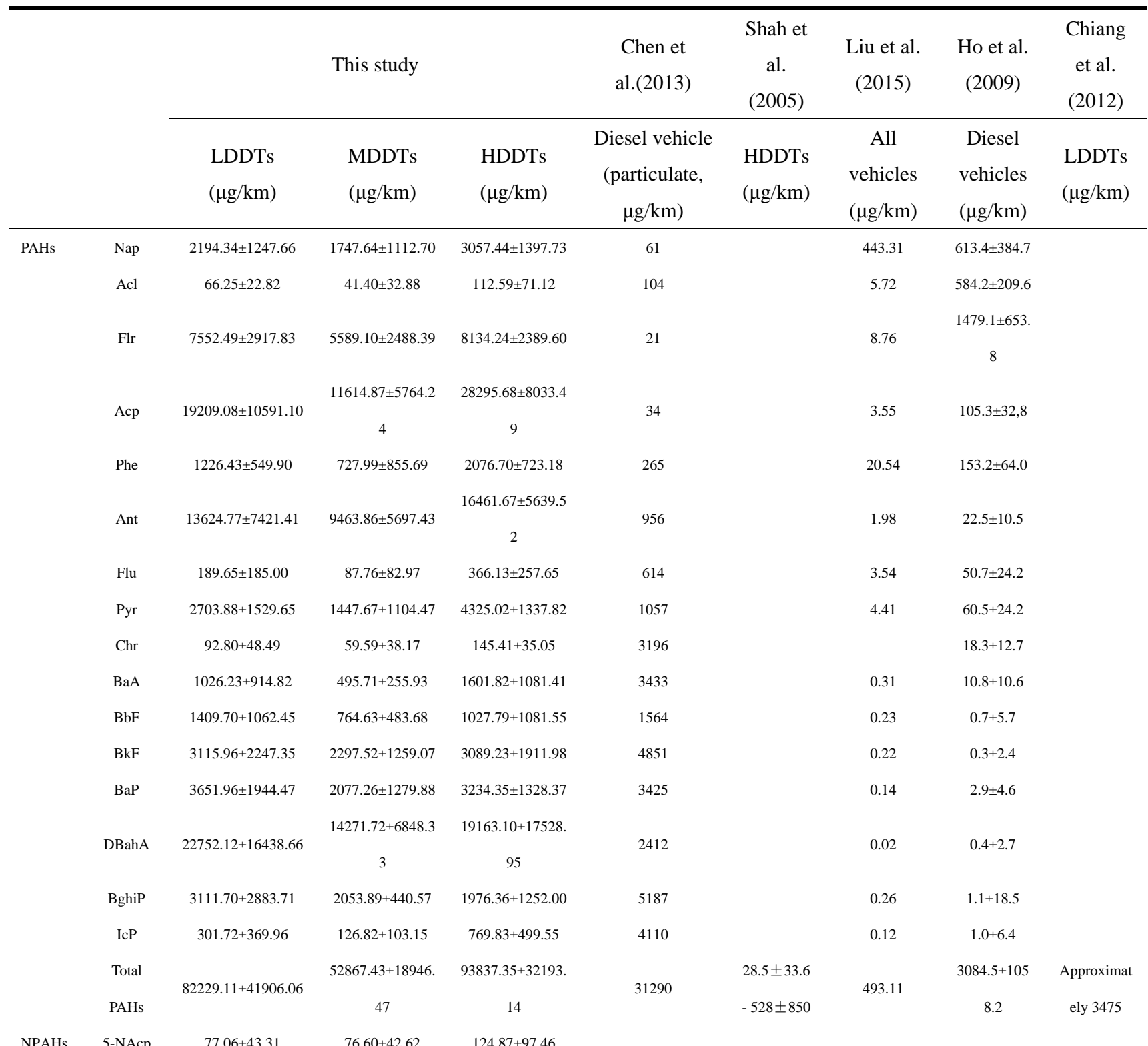




\begin{tabular}{cccc} 
9-NAnt & $4.76 \pm 3.44$ & $2.92 \pm 2.49$ & $5.81 \pm 2.49$ \\
3-NPhe & $720.41 \pm 389.25$ & $516.69 \pm 269.41$ & $861.37 \pm 325.91$ \\
1.3-NPyr & $48.68 \pm 24.63$ & $43.72 \pm 38.35$ & $96.22 \pm 44.44$ \\
1-NPyr & $135.66 \pm 46.75$ & $84.88 \pm 48.83$ & $184.65 \pm 84.11$ \\
3-NFlu & $38.87 \pm 25.01$ & $27.18 \pm 14.84$ & $47.65 \pm 19.71$ \\
6-NChr & $27.10 \pm 24.16$ & $23.81 \pm 19.52$ & $33.41 \pm 23.62$ \\
7-NBaA & $54.16 \pm 30.04$ & $30.61 \pm 22.89$ & $90.38 \pm 48.23$ \\
6-NBaP & $17.29 \pm 18.44$ & $35.96 \pm 39.83$ & $21.74 \pm 24.67$ \\
Total & & & \\
NPAHs & $1123.98 \pm 528.19$ & $842.38 \pm 345.70$ & $1466.09 \pm 481.48$ \\
\hline
\end{tabular}

Compared to the literature, the EFs for the total PAHs determined in this study are comparable to the results reported in Chen et al. (2013) and Shah et al. (2005). Chen et al. (2013) found that the EF of particle-phase PAHs from a diesel vehicle was $31.290 \mathrm{mg} / \mathrm{km}$ based on a tunnel test in Nanjing, China. Shah et al. (2005) determined that the EFs of the total PAHs for heavy-duty diesel vehicles under different driving patterns varied from $17.7 \pm 20.9$ to $328 \pm 528 \mathrm{mg} / \mathrm{km}$ based on on-road emission tests using CE-CERT's Mobile Emission Laboratory. However, large gaps were found compared with the total PAH EFs of $494 \mu \mathrm{g} / \mathrm{km}$ reported in Liu et al. (2015) and 3084.5 \pm 1058.2 $\mu \mathrm{g} / \mathrm{km}$ reported for the diesel vehicles tested in Ho et al. (2009) based on tunnel tests. The results determined in this study are also significantly higher than that found in Chiang et al. (2012), who reported that the total EF of PAHs plus NPAHs for light-duty diesel vehicles was $3.62 \mathrm{mg} / \mathrm{km}$ and the mass fraction of NPAHs was less than $4 \%$ based on dynamometer testing in a laboratory. These differences may arise from the vehicle technology, fuel, driving cycle, sampling method, analysis method, etc. More studies should be carried out to reduce the uncertainty of these results. 


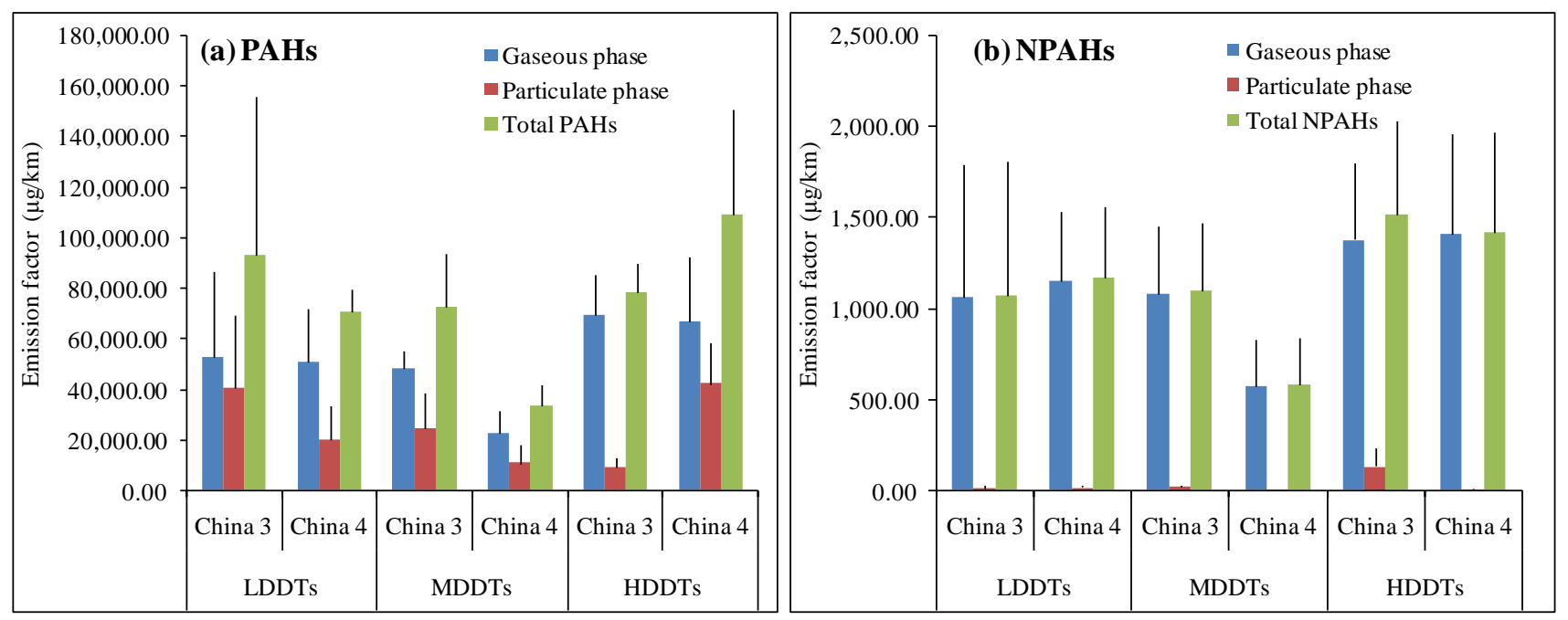

Figure 1 EFs of PAHs and NPAHs by vehicle type.

\subsection{Composition Characteristics}

Figure 2 demonstrates the gas- and particulate-phase distribution of PAHs and NPAHs for the different types of diesel trucks tested. A high mass share rate was found for gaseous-phase PAHs and NPAHs in the total PAHs and NPAHs. The gaseous-phase PAHs from different types of diesel trucks contributed $56 \%-89 \%$ of the total PAHs, with an average of $69 \%$. Regarding NPAHs, the gaseous phase accounted for approximately 97\%, varying from 91\%-100\%. The particulate-phase PAHs and NPAHs shared approximately $31 \%$ and $3 \%$ on average, respectively. A high share rate of gaseous PAHs was also found by Ho et al. (2009) who reported that the EFs of gaseous-phase PAHs were $~ 15$ times higher than particle-phase PAH EFs for all the vehicles in the Shing Mun Tunnel of Hong Kong. The gas-particle phase distribution for a diesel vehicle is related to the engine loading (Zielinska et al., 2004) and environmental temperature (Ho et al., 2009). Although the share rate of the gaseous-phase PAHs (40\%) reported for light-duty diesel vehicles determined by Chiang et al. (2012) was lower than that found in this study, the importance of the gaseous-phase PAHs is undeniable. However, existing studies have paid more attention to particulate-phase PAHs or NPAHs; thus, more attention should be paid to gaseous-phase PAHs due to their high contribution to the total emissions. 


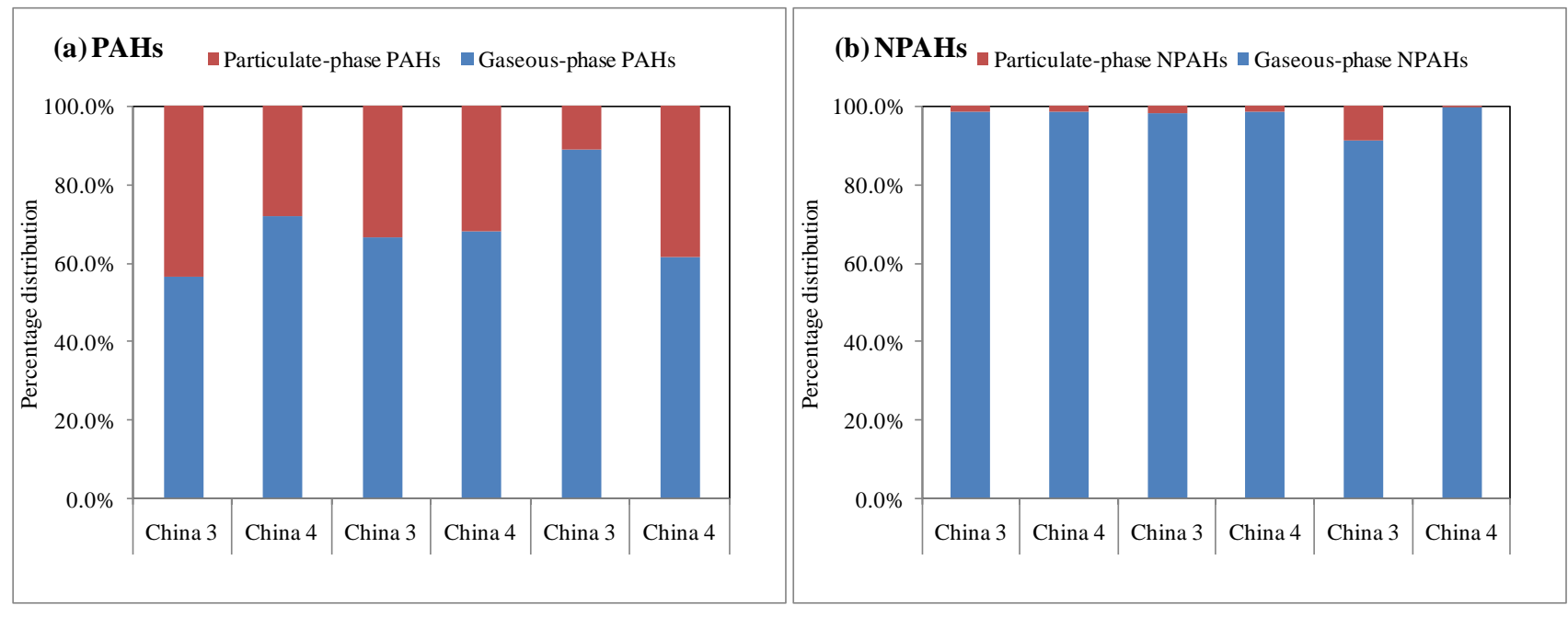

Figure 2 Distribution of gaseous- and particulate-phase PAHs and NPAHs from the tested diesel trucks by vehicle type.

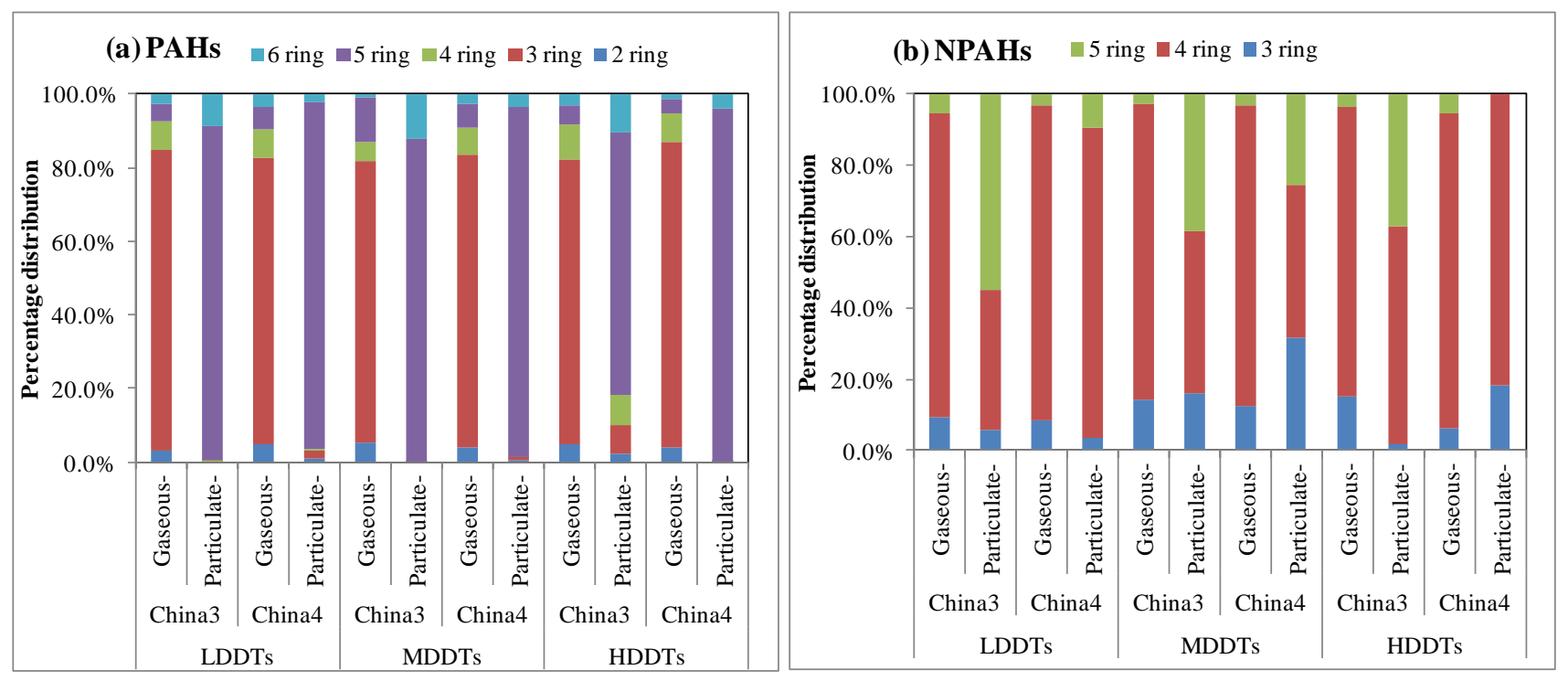

Figure 3 Percentage distribution of PAHs and NPAHs by the number of rings for the tested diesel trucks, on average.

Figure 3 shows the percentage distribution of PAHs and NPAHs by the number of rings for the different types of tested diesel trucks, on average. PAHs with three rings or less were the main contributors of the total gaseous-phase PAHs, contributing approximately $83.7 \%$ and ranging from $81.8 \%$ to $86.8 \%$, of which three-ring PAHs were the most abundant gaseous-phase PAHs with a share rate of 79.4\%. Particulate-phase PAHs mainly consisted of PAHs with five or more rings, 
reaching approximately $96.0 \%$ and varying from $81.7 \%$ to $99.9 \%$, of which five-ring PAHs were the most abundant particulate-phase PAHs, accounting for approximately $89.3 \%$. These results are very similar to the findings of other studies (Zielinska et al., 2004; Ho et al., 2009): low-molecular-weight PAHs were mainly in the gas phase, whereas PAHs with a greater number of rings were predominant in the particulate phase. The gaseous-phase NPAHs mainly consisted of three- and four-ring constituents, contributing approximately $95.9 \%$ on average, of which the four-ring constituents were most abundant with a mean contribution of approximately $84.9 \%$. The constituents of the particulate-phase NPAHs were dominated by five-ring NPAHs, especially China 3 diesel trucks.

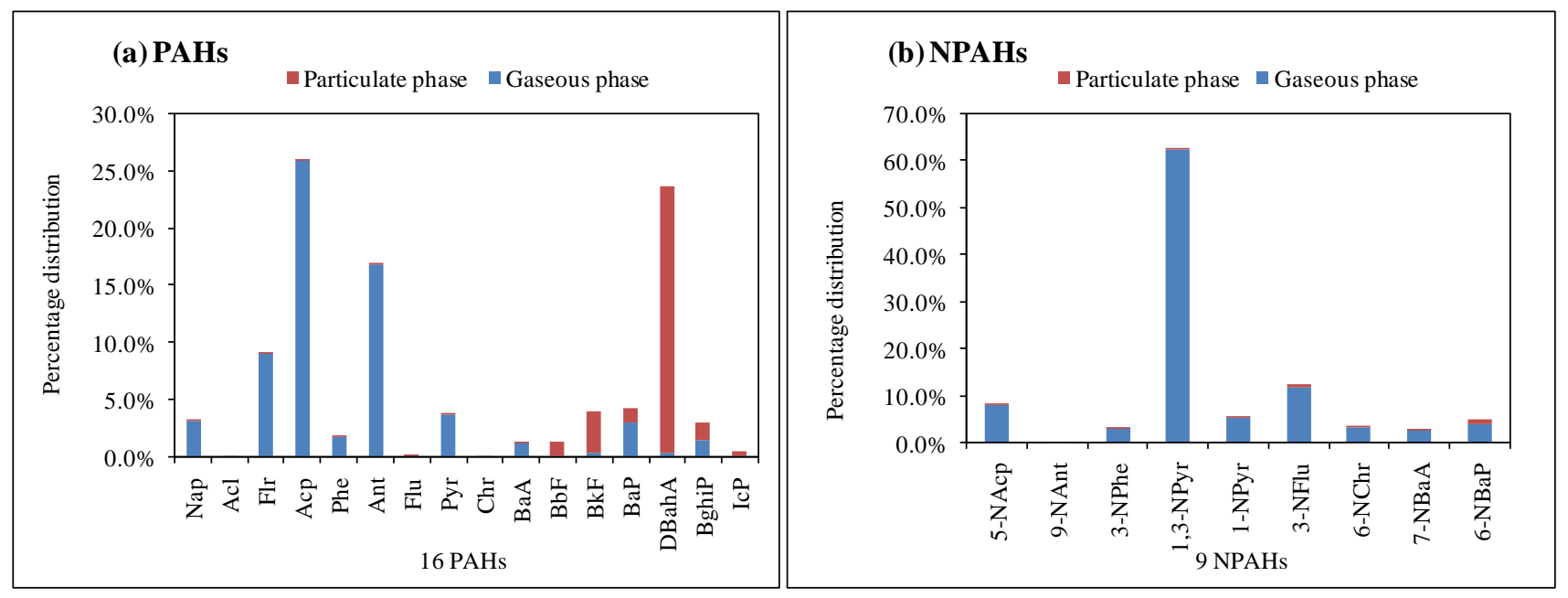

Figure 4 Percentage distribution of PAH and NPAH isomers from the tested diesel trucks, on average.

The percentage distributions of PAH and NPAH isomers from all the tested vehicles, on average, is shown in Figure 4. Acp, Ant and Flr were the three main gaseous-phase PAHs, with average percentages of approximately $26.1 \%, 16.9 \%$ and $9.2 \%$ contributing to the total PAHs, respectively. DBahA, BkF and BghiP were the most abundant particulate-phase PAHs, contributing approximately $23.2 \%, 3.7 \%$ and $1.5 \%$ of the total PAHs. Among the 16 examined PAH compounds, $\mathrm{BaP}$ is classified as a Group 1 carcinogen (IARC, 2012). The gaseous-phase and particulate-phase components of $\mathrm{BaP}$ constituted approximately $3.0 \%$ and $1.3 \%$ of the total PAHs, respectively. DBahA was originally classified as a "probable" human carcinogen (2A), whereas Nap, BaA, BbF, 
BkF and IcP were deemed "possible" human carcinogens (2B) (IARC, 2012).

1,3-NPyr, 3-NFlu and 5-NAcp were the three main gaseous-phase NPAHs, accounting for approximately $62.4 \%, 12.0 \%$ and $8.3 \%$ of the total PAHs, respectively. 6-NBap, 3-NFlu and 1,3-NPyr were the first three most abundant particulate-phase NPAHs, contributing approximately $0.9 \%, 0.5 \%$ and $0.4 \%$ of the total NPAHs. Although the NPAH emissions were significantly lower than those of their parent PAHs, NPAHs are of greater concern because some NPAHs have the potential for mutagenic and carcinogenic effects (Landvik et al., 2007).

\subsection{Influence of Driving Conditions}

Figure 5 presents the mean EFs of gaseous-phase, particulate-phase and total PAHs and NPAHs on highway and non-highway roads for the different types of tested diesel trucks, on average. For the LDDTs and HDDTs, most of the EFs of the gaseous-phase, particulate-phase and total PAHs and NPAHs on non-highway roads were higher than those on highways. In theory, more incomplete combustion will occur when diesel vehicles drive on non-highway roads, thus resulting in greater PAHs and NPAHs compared to that on highways. Shah et al. (2005). reported that the total PAH EFs of heavy-duty diesel vehicles during congested conditions are approximately an order of magnitude higher than during cruising conditions. Similar results were also reported for the diesel vehicles tested by Karavalakis et al., (2010). who also found lower PAH emissions under driving cycles at higher speeds because the increase in the average vehicle speed increases exhaust temperatures, thus resulting in better oxidation of these compounds. However, most of the EFs of gaseous-phase, particulate-phase and total PAHs and NPAHs for the MDDTs tested in this study presented opposite results with higher emissions on highways, similar to the abnormally lower EFs of PAHs and NPAHs compared to LDDTs and HDDTs. We could not determine an explanation for these results. 


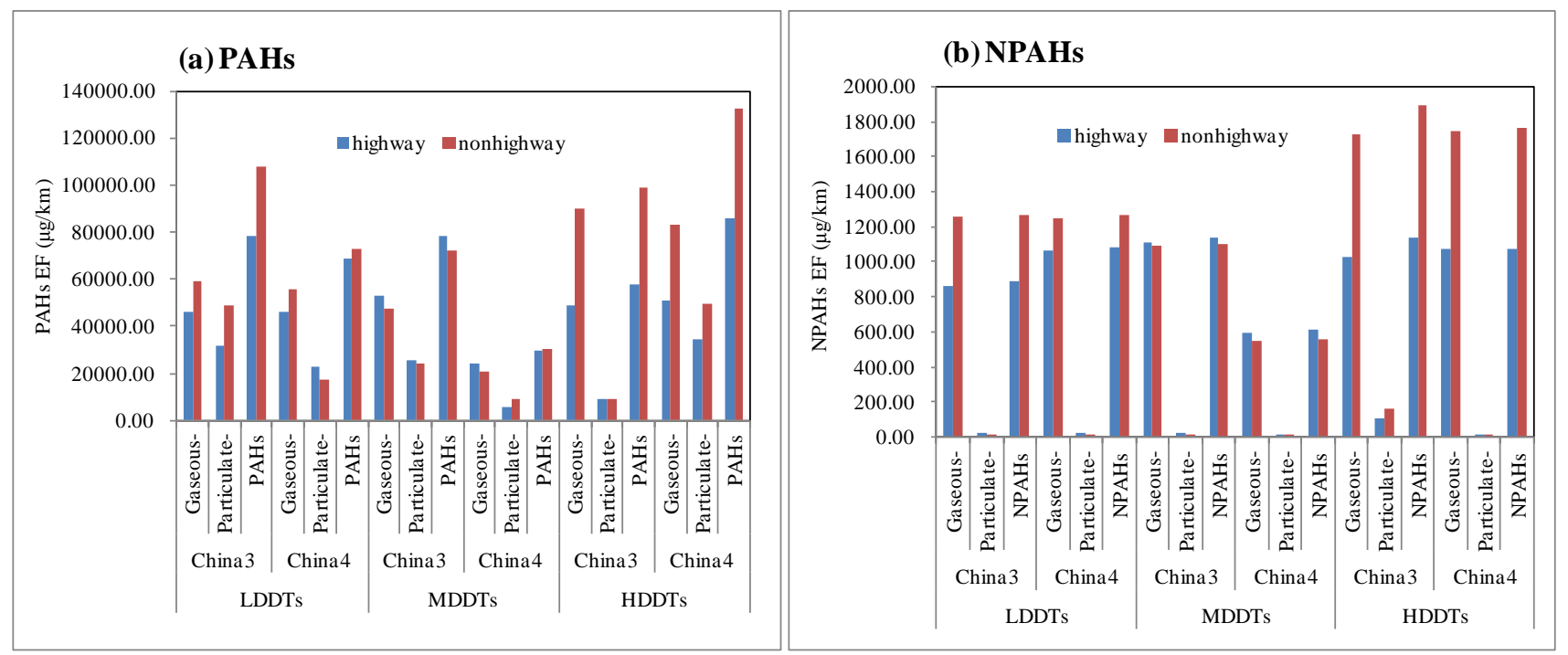

Figure 5 EFs of gaseous-phase, particulate-phase and total PAHs and NPAHs on highway and non-highway roads for the different types of tested diesel trucks, on average.

Figure S1 demonstrates the impacts of the road on the percentage distribution of gaseous- and particulate-phase PAHs relative to the number of rings for the tested diesel trucks, on average. The type of road had very slight impacts on the percentage distribution of gaseous-phase PAHs relative to the number of rings. The percentage distribution on highway and non-highway roads presented very similar results. However, the type of road had significant impacts on the particulate-phase PAHs with high numbers of rings, especially 6-ring particulate-phase PAHs. On average, the share rate of six-ring particulate-phase PAHs to the total particulate-phase PAHs changed from $4.3 \%$ to $10.6 \%$ with the change in the road from non-highway to highway.

The influence of the road on the percentage distribution of gaseous- and particulate-phase NPAHs relative to the number of rings was very similar to that of PAHs, as shown in Figure S2. The percentage distribution of gaseous-phase NPAHs did not greatly changed compared to that of particulate NPAHs with the change in the type of road. The driving patterns on highways results in more six-ring particulate-phase NPAHs. The contribution of six-ring constituents to the total particulate-phase PAHs on highways was approximately 50.9\%, which was 2.3 times that on non-highway roads.

\section{Conclusion}


This study attempted to understand the real emission characteristics of PAHs and NPAHs from diesel vehicles based on on-road emission measurements from 18 diesel trucks of different sizes and emission standards using a PEMS in Beijing, China. These results are helpful in understanding the emission levels and important impact factors of PAHs and NPAHs from diesel vehicles in China.

To control vehicle emissions, the Chinese government has implemented a series of measures. Regarding diesel vehicles, China 1, 2, 3 and 4 emission standards for new vehicles have been put in place in sequence. As a result, the EFs of $\mathrm{CO}, \mathrm{HC}$ and PM2.5 for diesel vehicles have greatly decreased with the strictness of the emission standards based on on-road emission measurement results. However, the existing emission standards only regulate the emission limits of $\mathrm{CO}, \mathrm{HC}, \mathrm{NOx}$ and PM2.5, not including PAHs, NPAHs, carbonyl compounds or other toxic pollutants. According to the results of this study, no decreasing trend was observed for PAH or NPAH emissions for the tested diesel trucks with China 3 to China 4 emission standards. Although the emission levels of PAHs and NPAHs were not high, the PAHs and NPAHs may be more harmful than the regulated pollutants due to their toxicity, mutagenicity and carcinogenicity. Thus, additional emission standards for PAHs and NPAHs for diesel vehicles should be regulated as soon as possible.

As described in the above section, the large gaps between the PAH and NPAH emissions from diesel vehicles reported in different studies should be noted. Even if the studies were carried out in the same county, the difference in the EFs of PAHs and NPAHs could reach several orders of magnitude. This large uncertainty may be related to the vehicle types, control technologies, driving cycles, fuel, sampling and analysis methods, etc. These uncertainties then would introduce errors in the development of a PAH and NPAH emission inventory. Thus, more tests, especially on NPAH emissions from diesel vehicles due to the limited reports and their higher toxicity than PAHs, should be performed to reduce these uncertainties. In addition, more attention should also be paid to gaseous-phase PAHs and NPAHs due to their high contribution to the total PAHs and NPAHs for diesel vehicles.

\section{Acknowledgements}

We thank Yunong Wu, Wei Zhang and Hui Wu for their assistance with the sampling and analysis of PAHs and NPAHs. This research was funded by the Natural Science Foundation of Beijing 
Municipality (8142011), the National Key Research and Development Program (2016YFC0201501), the National Science Foundation of China (41275124, 51278272) and the Research Ability Promotion Program for Graduate Student in 2016 at Beijing Technology and Business University.

\section{Appendix A. Supplementary data}

Detailed information on the tested diesel trucks is listed in Table S1; the average EFs of PAHs and NPAHs by vehicle type are presented in Table S2; the impacts of the type of road on the percentage distribution of gaseous- and particulate-phase PAHs relative to the number of rings for the tested diesel trucks, on average, are shown in Figure S1; the impacts of the type of road on the percentage distribution of gaseous- and particulate-phase NPAHs relative to the number of rings for the tested diesel trucks, on average, are shown in Figure S2.

\section{References}

Albinet, A., Leoz-Garziandia, E., Budzinski, H., Villenave, E., 2007. Polycyclic aromatic hydrocarbons (PAHs), nitrated PAHs and oxygenated PAHs in ambient air of the Marseilles area (south of France): concentrations and sources. Sci. Total Environ. 384 (1-3), 280-292. DOI 10.1016/j.scitotenv.2007.04.028.

Bamford, H. A., Baker, J. E., 2003. Nitro-polycyclic aromatic hydrocarbon concentrations and sources in urban and suburban atmospheres of the mid-Atlantic region. Atmos. Environ. 37 (15), 2077-2091. DOI 10.1016/S1352-2310(03)00102-X.

Benner, B. A., Gordon, G. E., Wise, S. A., 1989. Mobile sources of atmospheric polycyclic aromatic hydrocarbons: a roadway tunnel study. Environ. Sci. Technol. 23 (10), 1269-1278. DOI 10.1021/es00068a014.

Bikas, G., Zervas, E., 2007. Nonregulated pollutants emitted from euro 3 diesel vehicles as a function of their mileage. Energ. Fuels. 21 (5), 2731-2736. DOI 10.1021/ef070036d.

Casal, C. S., Arbilla, G., Corrêa, S. M., 2014. Alkyl polycyclic aromatic hydrocarbons emissions in diesel/biodiesel exhaust. Atmos. Environ. 96, 107-116. DOI 10.1016/j.atmosenv.2014.07.028.

Chang, K. F., Fang, G. C., Chen, J. C. ,Wu, Y. S., 2006. Atmospheric polycyclic aromatic hydrocarbons (PAHs) in Asia: a review from 1999 to 2004. Environ. Pollut. 142 (3), 388-396. DOI 10.1016/j.envpol.2005.09.025. 
Chen, B., Xuan, X., Zhu, L., Wang, J., Gao, Y., Yang, K., Shen, X., Lou, B., 2004. Distributions of polycyclic aromatic hydrocarbons in surface waters, sediments and soils of Hangzhou City, China. Water Res. 38 (16), 3558-3568. DOI 10.1016/j.watres.2004.05.013.

Chen, F., Hu, W., Zhong, Q., 2013. Emissions of particle-phase polycyclic aromatic hydrocarbons (PAHs) in the Fu Gui-shan tunnel of Nanjing, China. Atmos. Res. 124, 53-60. DOI 10.1016/j.atmosres.2012.12.008.

Chetwittayachan, T., Shimazaki, D., Yamamoto, K., 2002. A comparison of temporal variation of particle-bound polycyclic aromatic hydrocarbons (pPAHs) concentration in different urban environments: Tokyo, Japan, and Bangkok, Thailand. Atmos. Environ. 36 (12), 2027-2037. DOI 10.1016/S1352-2310(02)00099-7.

Chiang, H., Lai, Y., Chang, S., 2012. Pollutant constituents of exhaust emitted from light-duty diesel vehicles. Atmos. Environ. 47, 399-406. DOI 10.1016/j.atmosenv.2011.10.045.

Deabrantes, R., Deassuncao, J., Pesquero, C., 2004. Emission of polycyclic aromatic hydrocarbons from light-duty diesel vehicles exhaust. Atmos. Environ. 38 (11), 1631-1640. DOI 10.1016/j.atmosenv.2003.11.012.

Durant, J. L., Busby, W. F., Lafleur, A. L., Penman, B. W., Crespi, C. L., 1996. Human cell mutagenicity of oxygenated, nitrated and unsubstituted polycyclic aromatic hydrocarbons associated with urban aerosols. Mutat. Res. 371 (3-4), 123-157. DOI 10.1016/S0165-1218(96)90103-2.

Fraser, M. P., Cass, G. R., Simoneit, B. R. T., 1998. Gas-Phase and particle-phase organic compounds emitted from motor vehicle traffic in a Los Angeles roadway tunnel. Environ. Sci. Technol. 32 (14), 2051-2060. DOI 10.1021/es970916e.

Fromme, H., Lahrz, T., Piloty, M., Gebhardt, H., Oddoy, A., Rüden, H., 2004. Polycyclic aromatic hydrocarbons inside and outside of apartments in an urban area. Sci. Total Environ. 326 (1-3), 143-149. DOI 10.1016/j.scitotenv.2004.02.002.

Grimmer, G., Brune, H., Deutsch-Wenzel, R., Dettbarn, G., Jacob, J., Naujack, K. W., Mohr, U., Ernst, H., 1987. Contribution of polycyclic aromatic hydrocarbons and nitro-derivatives to the carcinogenic impact of diesel engine exhaust condensate evaluated by implantation into the lungs of rats. Cancer Lett. 37 (2), 173-180. DOI 10.1016/0304-3835(87)90160-1. 
Hao, X., Li, J., Yao, Z., 2016. Changes in PAHs levels in edible oils during deep-frying process. Food Control. 66, 233-240. DOI 10.1016/j.foodcont.2016.02.012.

Heeb, N. V., Schmid, P., Kohler, M., Gujer, E., Zennegg, M., Wenger, D., Wichser, A., Ulrich, A., Gfeller, U., Honegger, P., Zeyer, K., Emmenegger, L., Petermann, J. L., Czerwinski, J., Mosimann, T., Kasper, M., Mayer, A., 2010. Impact of low- and high-oxidation diesel particulate filters on genotoxic exhaust constituents. Environ. Sci. Technol. 44 (3), 1078-1084. DOI 10.1021/es9019222.

Ho, K. F., Ho, S. S. H., Lee, S. C., Cheng, Y., Chow, J. C., Watson, J. G., Louie, P. K. K., Tian, L., 2009. Emissions of gas- and particle-phase polycyclic aromatic hydrocarbons (PAHs) in the Shing Mun Tunnel, Hong Kong. Atmos. Environ. 43 (40), 6343-6351. DOI 10.1016/j.atmosenv.2009.09.025.

Hu, S., Herner, J. D., Robertson, W., Kobayashi, R., Chang, M. C., Huang, S. M., Zielinska, B., Kado, N., Collins, J. F., Rieger, P., Huai, T., Ayala, A., 2013. Emissions of polycyclic aromatic hydrocarbons (PAHs) and nitro-PAhs from heavy-duty diesel vehicles with DPF and SCR. J. Air Waste Manag. Assoc. 63 (8), 984-996. DOI 10.1080/10962247.2013.795202.

Huang, L., Bohac, S. V., Chernyak, S. M., Batterman, S. A., 2013. Composition and integrity of PAHs, nitro-PAhs, Hopanes, and Steranes in Diesel exhaust particulate matter. Water Air Soil Pollut. 224 (8), 1-14. DOI 10.1007/s11270-013-1630-1.

Huo, H., Yao, Z., Zhang, Y., Shen, X., Zhang, Q., He, K., 2012. On-board measurements of emissions from diesel trucks in five cities in china. Atmos. Environ. 54 (32), 159-167. DOI 10.1016/j.atmosenv.2012.01.068.

IARC, 2012. Monographs on the evaluation of carcinogenic risk to humans. In A Review of Human Carcinogens: Chemical Agents and Related Occupations vol 100F, International Arctic Research Center, University of Alaska, Fairbanks: Lyon, France.

Karavalakis, G., Deves, G., Fontaras, G., Stournas, S., Samaras, Z., Bakeas, E., 2010. The impact of soy-based biodiesel on PAH, nitro-PAH and oxy-PAH emissions from a passenger car operated over regulated and nonregulated driving cycles. Fuel. 89 (12), 3876-3883. DOI 10.1016/j.fuel.2010.07.002.

Landvik, N. E., Gorria, M., Arlt, V. M., Asare, N., Solhaug, A., Lagadic-Gossmann, D., Holme, J. A., 2007. Effects of nitrated-polycyclic aromatic hydrocarbons and diesel exhaust particle extracts on 
cell signalling related to apoptosis: possible implications for their mutagenic and carcinogenic effects. Toxicol. 231 (2-3), 159-174. DOI 10.1016/j.tox.2006.12.009.

Liu, Y., Gao, Y., Yu, N., ZHang, C., Wang, S., Ma, L., Zhao, J., Lohmann, R., 2015. Particulate matter, gaseous and particulate polycyclic aromatic hydrocarbons (PAHs) in an urban traffic tunnel of China: emission from on-road vehicles and gas-particle partitioning. Chemosphere. 134, 52-59. DOI 10.1016/j.chemosphere.2015.03.065.

Logan, D. T., 2007. Perspective on ecotoxicology of PAHs to fish. Human and ecological risk assessment. An. Int. J. 13 (2), 302-316.

Ravindra, K., Bencs, L., Wauters, E., de Hoog, J., Deutsch, F., Roekens, E., Bleux, N., Berghmans, P., Van Grieken, R., 2006. Seasonal and site-specific variation in vapour and aerosol phase PAHs over Flanders (Belgium) and their relation with anthropogenic activities. Atmos. Environ. 40 (4), 771-785. DOI 10.1016/j.atmosenv.2005.10.011.

Shah, S. D., Ogunyoku, T. A., Miller, J. W., Cocker III, D. R., 2005. On-road emission rates of PAH and n-Alkane compounds from heavy-duty diesel vehicles. Environ. Sci. Technol. 39 (14), $5276-5284$.

Shen, G., Tao, S., Wei, S., Zhang, Y., Wang, R., Wang, B., Li, W., Shen, H., Huang, Y., Chen, Y., Chen, H., Yang, Y., Wang, W., Wang, X., Liu, W., Simonich, S. L., 2012. Emissions of parent, nitro, and oxygenated polycyclic aromatic hydrocarbons from Residential Wood combustion in Rural China. Environ. Sci. Technol. 46 (15), 8123-8130. DOI 10.1021/es301146v.

Shen, H., Huang, Y., Wang, R., Zhu, D., Li., W., Shen, G., Wang, B., Zhang, Y., Chen, Y., Lu, Y., Chen, H., Li, T., Sun, K., Li, B., Liu, W., Liu, J., Tao, S., 2013. Global atmospheric emissions of polycyclic aromatic hydrocarbons from 1960 to 2008 and future predictions. Environ. Sci. Technol. 47 (12), 6415-6424. DOI 10.1021/es400857z.

Thuy, P. C., Kameda, T., Toriba, A., Tang, N., Hayakawa, K., 2012. Characteristics of atmospheric polycyclic aromatic hydrocarbons and Nitropolycyclic aromatic hydrocarbons in Hanoi-Vietnam, as a typical Motorbike City. Polycyclic Aromat. Compd. 32 (2), 296-312. DOI 10.1080/10406638.2012.679015.

Tsapakis, M., Stephanou, E. G., 2007. Diurnal Cycle of PAHs, Nitro-PAHs, and oxy-PAHs in a High Oxidation Capacity Marine Background Atmosphere. Environ. Sci. Technol. 41 (23), 8011-8017. DOI 10.1021/es071160e. 
Wada, M., Kido, H., Kishikawa, N., Tou, T., Tanaka, M., Tsubokura, J., Shironita, M., Matsui, M., Kuroda, N., Nakashima, K., 2001. Assessment of air pollution in Nagasaki city: determination of polycyclic aromatic hydrocarbons and their nitrated derivatives, and some metals. Environ. Pollut. 115 (1), 139-147. DOI 10.1016/S0269-7491(01)00093-8.

Wu, B., Shen, X., Cao, X., Yao, Z., Wu, Y., 2016. Characterization of the chemical composition of PM2.5 emitted from on-road china III and china IV diesel trucks in Beijing, China. Sci. Total Environ. 551-552, 579-589. DOI 10.1016/j.scitotenv.2016.02.048.

Yao, Z., Huo, H., Zhang, Q., Streets, D. G., He, K., 2011. Gaseous and particulate emissions from rural vehicles in china. Atmos. Environ. 45 (18), 3055-3061. DOI 10.1016/j.atmosenv.2011.03.012.

Yao, Z., Li, J., Wu, B., Hao, X., Yin, Y., Jiang, X., 2015a. Characteristics of PAHs from deep-frying and frying cooking fumes. Environ. Sci. Pollut. Res. Int. 22 (20), 16110-16120. DOI 10.1007/s11356-015-4837-4.

Yao, Z., Shen, X., Ye, Y., Cao, X., Jiang, X., Zhang, Y., He, K., 2015b. On-road emission characteristics of VOCs from diesel trucks in Beijing, China. Atmos. Environ. 103 (103), 87-93. DOI 10.1016/j.atmosenv.2014.12.028.

Yao, Z., Wu, B., Wu, Y., Cao, X., Jiang, X., 2015c. Comparison of NOx emissions from china iii and china iv in-use diesel trucks based on on-road measurements. Atmos. Environ. 123, 1-8. DOI 10.1016/j.atmosenv.2015.10.056.

Zhang, S., Zhang, W., Wang, K., Shen, Y., Hu, L., Wang, X., 2009. Concentration, distribution and source apportionment of atmospheric polycyclic aromatic hydrocarbons in the southeast suburb of Beijing, China. Environ. Monit. Assess. 151 (1-4), 197-207. DOI 10.1007/s10661-008-0261-2.

Zhou, J., Wang, T., Huang, Y., Mao, T., Zhong, N., 2005. Size distribution of polycyclic aromatic hydrocarbons in urban and suburban sites of Beijing, China. Chemosphere. 61 (6), 792-799. DOI 10.1016/j.chemosphere.2005.04.002.

Zielinska, B., Sagebiel, J., Arnott, W. P., Rogers, C. F., Kelly, K. E., Wagner, D. A., Lighty, J. S., Sarofim, A. F., Palmer, G., 2004. Phase and size distribution of polycyclic aromatic hydrocarbons in diesel and gasoline vehicle emissions. Environ. Sci. Technol. 38 (9), 2557-2567. DOI 10.1021/es030518d. 